University of Nebraska - Lincoln

DigitalCommons@University of Nebraska - Lincoln

2006

\title{
Morphology and Tensile Properties of Multigraft Copolymers with Regularly Spaced Tri-, Tetra-, and Hexafunctional Junction Points
}

Yuqing Zhu

Department of Polymer Science \& Engineering, UniVersity of Massachusetts, Amherst, Massachusetts

Engin Burgaz

Department of Polymer Science \& Engineering, UniVersity of Massachusetts, Amherst, Massachusetts

Samuel P. Gido

Department of Polymer Science \& Engineering, UniVersity of Massachusetts, Amherst, Massachusetts

Ulrike Staudinger

Leibniz Institute of Polymer Research Dresden (IPF), Hohe Strasse 6, 01069 Dresden, Germany

Roland Weidisch

Leibniz Institute of Polymer Research Dresden (IPF), Hohe Strasse 6, 01069 Dresden, Germany

See next page for additional authors

Follow this and additional works at: https://digitalcommons.unl.edu/usdoepub

Part of the Bioresource and Agricultural Engineering Commons

Zhu, Yuqing; Burgaz, Engin; Gido, Samuel P.; Staudinger, Ulrike; Weidisch, Roland; Uhrig, David; and Mays, Jimmy W., "Morphology and Tensile Properties of Multigraft Copolymers with Regularly Spaced Tri-, Tetra-, and Hexafunctional Junction Points" (2006). US Department of Energy Publications. 56.

https://digitalcommons.unl.edu/usdoepub/56

This Article is brought to you for free and open access by the U.S. Department of Energy at DigitalCommons@University of Nebraska - Lincoln. It has been accepted for inclusion in US Department of Energy Publications by an authorized administrator of DigitalCommons@University of Nebraska - Lincoln. 


\section{Authors}

Yuqing Zhu, Engin Burgaz, Samuel P. Gido, Ulrike Staudinger, Roland Weidisch, David Uhrig, and Jimmy W. Mays 


\title{
Morphology and Tensile Properties of Multigraft Copolymers with Regularly Spaced Tri-, Tetra-, and Hexafunctional Junction Points
}

\author{
Yuqing Zhu, ${ }^{\dagger}$ Engin Burgaz, and Samuel P. Gido* \\ Department of Polymer Science \& Engineering, University of Massachusetts, \\ Amherst, Massachusetts 01003
}

\section{Ulrike Staudinger and Roland Weidisch*,*}

Leibniz. Institute of Polymer Research Dresden (IPF), Hohe Strasse 6, 01069 Dresden, Germany

\author{
David Uhrig and Jimmy W. Mays ${ }^{\S}$ \\ Chemical Sciences Division and Center for Nanophase Materials Sciences, Oak Ridge National \\ Laboratory, Oak Ridge, Tennessee 37831
}

Received January 11, 2006; Revised Manuscript Received April 19, 2006

\begin{abstract}
The effect of chain architecture on the morphological and tensile properties of series of multigraft copolymers, with regularly spaced tri-, tetra-, and hexafunctional junction points, was investigated using transmission electron microscopy (TEM), small-angle X-ray scattering (SAXS), and tensile testing. The materials were synthesized by coupling difunctional polyisoprene (PI) spacers and living polystyrene (PS) branches, made by anionic polymerization, with chlorosilanes of different functionalities. Since the coupling process is a step-growth polymerization, yielding polydisperse products, fractionation was utilized to separate each material into three fractions (high, middle, and low molecular weight), each of low polydispersity. All three fractions have the same chain architecture on a per junction point basis but differ in the number of junction point units per molecule. By applying the constituting block copolymer concept, the physical behavior of these molecules was compared with current theories. It was found that morphological behavior of these graft copolymers can be predicted using theoretical approaches and is independent on the number of junction points. The number of the junction points, however, greatly influences the long-range order of microphase separation. Additionally, two new parameters for adjusting mechanical properties of multigraft copolymers were found in this investigation: (1) functionality of the graft copolymer-tri-, tetra-, or hexafunctional-and (2) number of junction points per molecule. An increase in functionality causes a change in morphology, resulting in a high level of tensile strength for tetrafunctional (cylindrical) and hexafunctional (lamellae) multigraft copolymers, leading to about the twice the strength of the spherical trifunctional multigrafts of similar overall composition. Tetrafunctional multigraft copolymers show a surprisingly high strain at break, far exceeding that of commercial block copolymer thermoplastic elastomers (TPEs). Strain at break and tensile strength increase linearly with the number of junction points per molecule. Hysteresis experiments at about 300-900\% deformation demonstrate that multifunctional multigraft copolymers have improved high elasticity as compared to commercial TPEs like Kraton or Styroflex.
\end{abstract}

\section{Introduction}

Block copolymer molecular architecture has been long recognized as an important factor for tailoring material properties. Changing from simple linear diblock copolymers to graft copolymers can greatly alter the dependence of the morphologies on the component volume fractions. ${ }^{1-11}$ It has been shown that complex graft copolymer architectures with multiple grafting points (Figure 1) can be understood morphologically by analogy to fundamental building blocks defined as the average structure per junction point. ${ }^{12,13}$ This fundamental component of a larger graft molecular architecture is referred to as the constituting block copolymer. ${ }^{9-15}$ For a graft copolymer with a backbone of $\mathrm{A}$ and blocks of $\mathrm{B}$ joined to the backbone at trifunctional junction points (Figure 1a), the constituting block copolymer

\footnotetext{
† Present address: Eastman Chemical Company, Kingsport, TN 37662. $\$$ Present address: Institute of Materials Science and Technology, Friedrich-Schiller-University Jena, Löbdergraben 32, 07743 Jena, Germany.

$\S$ Also: Department of Chemistry, University of Tennessee, Knoxville, TN 37996

*Corresponding authors: gido@mail.pse.umass.edu; roland.weidisch@ uni-jena.de.
}

(a)

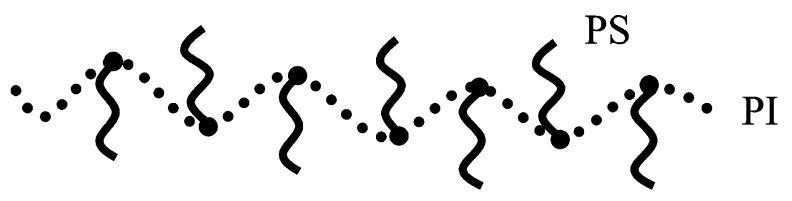

(b)

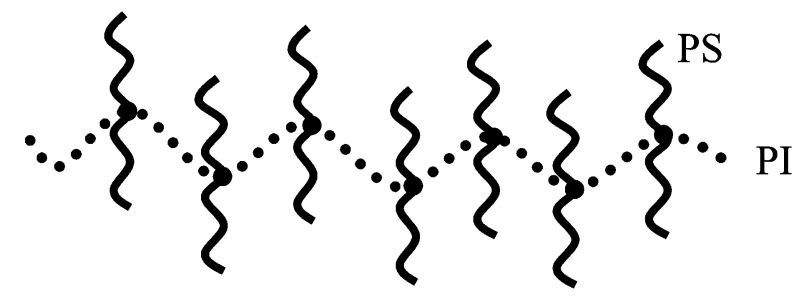

(c)

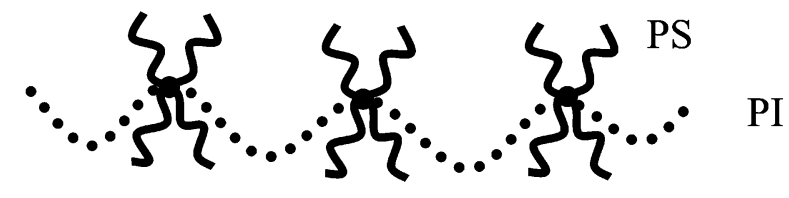

Figure 1. Illustration of multigraft copolymers based on PI backbone and PS branches with regularly spaced (a) trifunctional, (b) tetrafunctional, and (c) hexafunctional junction points. 
Table 1. Molecular and Morphological Characterization of Multigraft Copolymers with Regularly Spaced Trifunctional Junction Points

\begin{tabular}{|c|c|c|c|c|c|c|}
\hline & & $M_{\mathrm{w}}{ }^{a}\left(\times 10^{3} \mathrm{~g} / \mathrm{mol}\right)$ & no. of junctions/molecule & PS $(\operatorname{vol} \%)^{b}$ & morphology & $D^{\mathrm{c}}(\mathrm{nm})$ \\
\hline \multirow[t]{2}{*}{ MG-3-5 } & PS branch & 9.5 & & & & \\
\hline & PI spacer & 105 & & & & \\
\hline MG-3-5-4.3 & & 601 & 4.3 & 6.0 & disordered & \\
\hline MG-3-5-2.6 & & 406 & 2.6 & 5.9 & disordered & \\
\hline MG-3-5-1.8 & & 316 & 1.8 & 5.8 & disordered & \\
\hline \multirow[t]{2}{*}{ MG-3-17 } & PS branch & 31.6 & & & & \\
\hline & PI spacer & 86 & & & & \\
\hline MG-3-17-3.7 & & 515 & 3.7 & 17.2 & PS spheres & 40.3 \\
\hline MG-3-17-2.6 & & 391 & 2.6 & 17.5 & PS spheres & 42.2 \\
\hline MG-3-17-2.0 & & 315 & 2.0 & 17.8 & PS spheres & 41.0 \\
\hline \multirow[t]{2}{*}{ MG-3-43 } & PS branch & 60.3 & & & & \\
\hline & PI spacer & 48.0 & & & & \\
\hline MG-3-44-3.8 & & 464 & 3.8 & 41.6 & lamellae & 59.1 \\
\hline MG-3-44-3.3 & & 400 & 3.3 & 44.5 & lamellae & 59.3 \\
\hline MG-3-44-2.9 & & 360 & 2.9 & 45.9 & lamellae & 57.6 \\
\hline \multirow[t]{2}{*}{ MG-3-64 } & PS branch & 77.3 & & & & \\
\hline & PI spacer & 34.2 & & & & \\
\hline MG-3-64-3.5 & & 412 & 3.5 & 62.2 & not-ordered lamellae & 55.6 \\
\hline MG-3-64-2.7 & & 388 & 2.7 & 64.1 & lamellae & 54.8 \\
\hline MG-3-64-2.1 & & 268 & 2.1 & 64.5 & lamellae & 57.6 \\
\hline \multirow[t]{2}{*}{ MG-3-80 } & PS branch & 79.5 & & & & \\
\hline & PI spacer & 23.6 & & & & \\
\hline MG-3-80-3.5 & & 382 & 3.5 & 79.8 & PI cylinders & 41.0 \\
\hline MG-3-80-2.6 & & 291 & 2.6 & 80.1 & PI cylinders & 41.0 \\
\hline
\end{tabular}

${ }^{a}$ Weight-average molecular weight measured using SEC-MALLS (multiangle laser light scattering). ${ }^{b}$ PS volume percent calculated based on the averaged mass percentage from ${ }^{1} \mathrm{H}$ NMR and SEC-UV analyses. ${ }^{c}$ Domain spacing $D=2 \pi / q^{*}$, where $q^{*}$ is the primary Bragg's reflection.

is an $\mathrm{A}_{2} \mathrm{~B}$ single graft copolymer. Thus, the whole molecule can be viewed as a string of several such constituting units. The number of junction points per molecule is the same as the number of the constituting block copolymer units. The constituting unit of the regular graft copolymers that are the focus of this study can be described by a general formula $\mathrm{A}_{2} \mathrm{~B}_{m}$, where $\mathrm{A}$ are blocks of backbone material equal to half of each of the two difunctional connectors attached to each junction point. At each junction point, $m$ B blocks are grafted to the A backbone.

It has been found that the morphological behavior of regular multigraft copolymers with multiple trifunctional or tetrafunctional junction points is generally consistent with the morphologies of their constituting block copolymers. ${ }^{1,4,9-13}$ However, the long-range order that can be achieved is diminished as the number of junction points per molecule $(n)$ increases. ${ }^{13}$ Preliminary studies of the tensile properties of multigraft copolymer, thermoplastic elastomers with regularly spaced tetrafunctional junction points, showed that they support significantly higher elongation than commercial materials while achieving comparable stress at break. ${ }^{16}$ It was also found that the stress at break and the strain at break in these multigraft materials increased with increasing number of the junction points.

The present study concentrates on the effect of chain architecture on the morphologies and tensile properties of three series of multigraft copolymers: one with trifunctional, one with tetrafunctional, and one with hexafunctional junction points. All three series have polyisoprene (PI) backbones and polystyrene (PS) grafts. The PS volume fractions for each series cover the whole range of the morphological diagram. Figure 1 illustrates these three types of molecular architectures. The copolymers were synthesized in a step-growth macromonomer polymerization by connecting near-monodisperse living PS branches and difunctional PI spacers in the presence of chlorosilane coupling agents. ${ }^{17}$ The resulting materials are polydisperse in the number of junction points (constituting units) per molecule, but the local structure of each constituting unit is much less polydisperse, reflecting the anionic synthesis of the component blocks. Solvent - nonsolvent fractionation was employed so that each material is subdivided into three fractions, all of which have relatively narrower polydispersity $(<1.2)$.

\section{Experimental Section}

Synthesis and Molecular Characterization. Details of the synthesis and molecular characterization of these multigraft copolymers can be found elsewhere. ${ }^{17}$ Molecular characteristics of the multigraft copolymers with trifunctional, tetrafunctional, and hexafunctional junction points, the PS branches, and the PI spacers are listed in Tables 1-3. Each as-synthesized material was divided into low, middle, and high molecular weight fractions by solventnonsolvent fractionation. A general nomenclature for these multigraft copolymers, MG- $n-\phi-\beta$, is employed. MG stands for "regular multigraft", and $n$ is the junction point functionality. The " $\phi$ " represents the total PS volume percent, and the " $\beta$ " refers to the averaged number of junction points per molecule in a given fraction, which is sometimes omitted to represent all three fractions in the same material. Thus, MG-6-21-5.2 refers to the fraction of the regular multigraft copolymer (MG) with hexafunctional junction points, a PS volume percent of 21, and an average of 5.2 junction points per molecule.

Morphological Characterization and Tensile Testing. $3 \mathrm{wt}$ $\%$ solutions of block copolymer in toluene were prepared, and bulk films about $0.2-0.3 \mathrm{~mm}$ thick were obtained by slowly evaporating the solvent from these solutions over 14 days at room temperature. The dried films were then annealed at $120^{\circ} \mathrm{C}$ under vacuum for 7 days to further equilibrate the morphologies. All samples for transmission electron microscopy (TEM) were microtomed using a Leica Ultracut UCT cryo-ultramicrotome. Thin sections 40-80 $\mathrm{nm}$ in thickness were cut using a Diatome diamond knife at a sample temperature of $-110^{\circ} \mathrm{C}$ and a knife temperature of $-100{ }^{\circ} \mathrm{C}$. These sections were collected on TEM grids and stained in $\mathrm{OsO}_{4}$ vapor for $4 \mathrm{~h}$. TEM was performed on a JEOL $100 \mathrm{CX}$, operated at an accelerating voltage of $100 \mathrm{kV}$.

Small-angle X-ray scattering (SAXS) data were obtained using Ni-filtered $\mathrm{Cu} \mathrm{K} \alpha$ radiation (1.54 $\AA$ wavelength) from a Rigaku rotating anode operated at $40 \mathrm{kV}, 200 \mathrm{~mA}$. The primary beam was collimated by a set of three pinholes. A gas-filled area detector (Siemens Hi-Star), located $87.52 \mathrm{~cm}$ from the sample, was used to record scattering patterns. The flight path between the sample and the detector was evacuated. The two-dimensional scattering patterns were circularly averaged to give the $(\log I)$ vs $q$ plots, where $I$ is the scattering intensity and $q$ is the scattering vector. 
Table 2. Molecular and Morphological Characterization of Multigraft Copolymers with Regularly Spaced Tetrafunctional Junction Points

\begin{tabular}{|c|c|c|c|c|c|c|}
\hline & & $M_{\mathrm{w}}{ }^{a}\left(\times 10^{3} \mathrm{~g} / \mathrm{mol}\right)$ & no. of junctions/molecule & PS $(\operatorname{vol} \%)^{b}$ & morphology & $D^{\mathrm{c}}(\mathrm{nm})$ \\
\hline MG-4-14 & $\begin{array}{l}\text { PS branch } \\
\text { PI spacer }\end{array}$ & $\begin{array}{l}11.1 \\
97.2\end{array}$ & & & & \\
\hline MG-4-14-5.5 & & 691 & 5.5 & 14.2 & PS spheres & 28.5 \\
\hline MG-4-14-3.5 & & 475 & 3.5 & 14.2 & PS spheres & 28.5 \\
\hline MG-4-14-2.5 & & 372 & 2.5 & 14.0 & PS spheres & 31.5 \\
\hline MG-4-23 & $\begin{array}{l}\text { PS branch } \\
\text { PI spacer }\end{array}$ & $\begin{array}{l}16.8 \\
87.6\end{array}$ & & & & \\
\hline MG-4-23-6.6 & & 891 & 6.6 & 23.3 & PS cylinders & 31.5 \\
\hline MG-4-23-4.4 & & 624 & 4.4 & 22.8 & PS cylinders & 31.5 \\
\hline MG-4-23-3.2 & & 472 & 3.2 & 22.7 & PS cylinders & 33.9 \\
\hline MG-4-41 & $\begin{array}{l}\text { PS branch } \\
\text { PI spacer }\end{array}$ & $\begin{array}{l}26.6 \\
63.6\end{array}$ & & & & \\
\hline MG-4-41-7.4 & & 930 & 7.4 & 40.8 & lamellae & 38.4 \\
\hline MG-4-41-5.3 & & 681 & 5.3 & 41.0 & lamellae & 38.4 \\
\hline MG-4-41-4.4 & & 572 & 4.4 & 41.0 & lamellae & 41.0 \\
\hline
\end{tabular}

${ }^{a}$ Weight-average molecular weight measured using SEC-MALLS (multiangle laser light scattering). ${ }^{b}$ PS volume percent calculated based on the averaged mass percentage from ${ }^{1} \mathrm{H}$ NMR and SEC-UV analyses. ${ }^{c}$ Domain spacing $D=2 \pi / q^{*}$, where $q^{*}$ is the primary Bragg's reflection.

Table 3. Molecular and Morphological Characterization of Multigraft Copolymers with Regularly Spaced Hexafunctional Junction Points

\begin{tabular}{|c|c|c|c|c|c|c|}
\hline & & $M_{\mathrm{w}}{ }^{a}\left(\times 10^{3} \mathrm{~g} / \mathrm{mol}\right)$ & no. of junctions/molecule & PS $(\operatorname{vol} \%)^{b}$ & morphology & $D^{\mathrm{c}}(\mathrm{nm})$ \\
\hline \multirow[t]{2}{*}{ MG-6-21 } & PS branch & 8.2 & & & & \\
\hline & PI spacer & 86.8 & & & & \\
\hline MG-6-21-5.2 & & 705 & 5.2 & 21.8 & microphase separated & 34.6 \\
\hline MG-6-21-3.6 & & 515 & 3.6 & 21.6 & lamellae & 34.6 \\
\hline MG-6-21-2.7 & & 411 & 2.7 & 21.4 & lamellae & 34.6 \\
\hline \multirow[t]{2}{*}{ MG-6-35 } & PS branch & 13.0 & & & & \\
\hline & PI spacer & 63.3 & & & & \\
\hline MG-6-35-3.0 & & 409 & 3.0 & 35.6 & lamellae & 46.4 \\
\hline MG-6-35-2.3 & & 328 & 2.3 & 35.4 & lamellae & 44.1 \\
\hline MG-6-35-1.9 & & 287 & 1.9 & 35.6 & lamellae & 38.4 \\
\hline
\end{tabular}

${ }^{a}$ Weight-average molecular weight measured using SEC-MALLS (multiangle laser light scattering). ${ }^{b}$ PS volume percent calculated based on the averaged mass percentage from ${ }^{1} \mathrm{H}$ NMR and SEC-UV analyses. ${ }^{c}$ Domain spacing $D=2 \pi / q^{*}$, where $q^{*}$ is the primary Bragg's reflection.

The assignment of a morphological structure, spheres, cylinders, etc., was based upon the combined evidence of the TEM and SAXS data. Especially for samples with higher numbers of branch points per molecule, the long-range order of the morphology was often suppressed. In these cases the TEM images were examined for clues as to the shape of the microphase-separated domains; i.e., are they compact like spheres, extended like cylinders, or repeatedly branching as in a bicontinuous structure? In these disordered cases the SAXS data typically lack Bragg peaks, but the broad maxima present were compared with form factor scattering, for instance from spheres and cylinders, to aid in morphology identification.

Tensile tests were performed at a cross-head speed of 15 $\mathrm{mm} / \mathrm{min}$ at room temperature using a universal testing machine (Zwick 1456) according to ISO 527 (specimen type 5A). To ensure good measurement statistics, 5-10 tensile specimens were prepared for each sample by stamping the dog-bone shape from cast and annealed polymer films. To accurately measure the sample strain, mechanical Multisens equipment was used to measure the elongation in the gauge length only. This is in contrast to the simpler, but less accurate, method of equating strain to the tensile tester cross-head displacement. From this elongation of the specimens in the gauge length and applied load during test, engineering strain $(\epsilon)$ and engineering stress $(\sigma)$ have been calculated (ISO 527).

\section{Results and Discussion}

Morphology. Figure 2 displays a series of images for the three fractions of MG-6-21, where long-range order increases with decreasing average number of junction points per molecule. In MG-6-21-5.2, which has an average of 5.2 junction points per molecule, the material is microphase-separated without any long-range order, as shown in Figure 2a. Some poorly ordered lamellae were found in MG-6-21-3.6, as those shown in Figure 2b. Shown in Figure 2c is the MG-6-21-2.7 with 2.7 junction
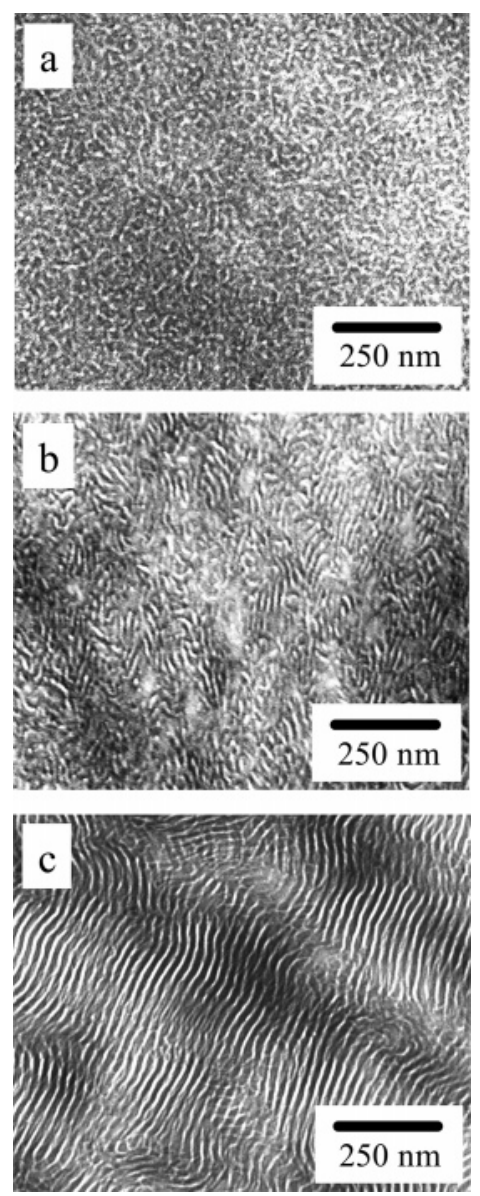

Figure 2. TEM images of hexafunctional multigraft copolymers with 21\% of PS: (a) MG-6-21-5.3, (b) MG-6-21-3.6, and (c) MG-6-21-2.7. 

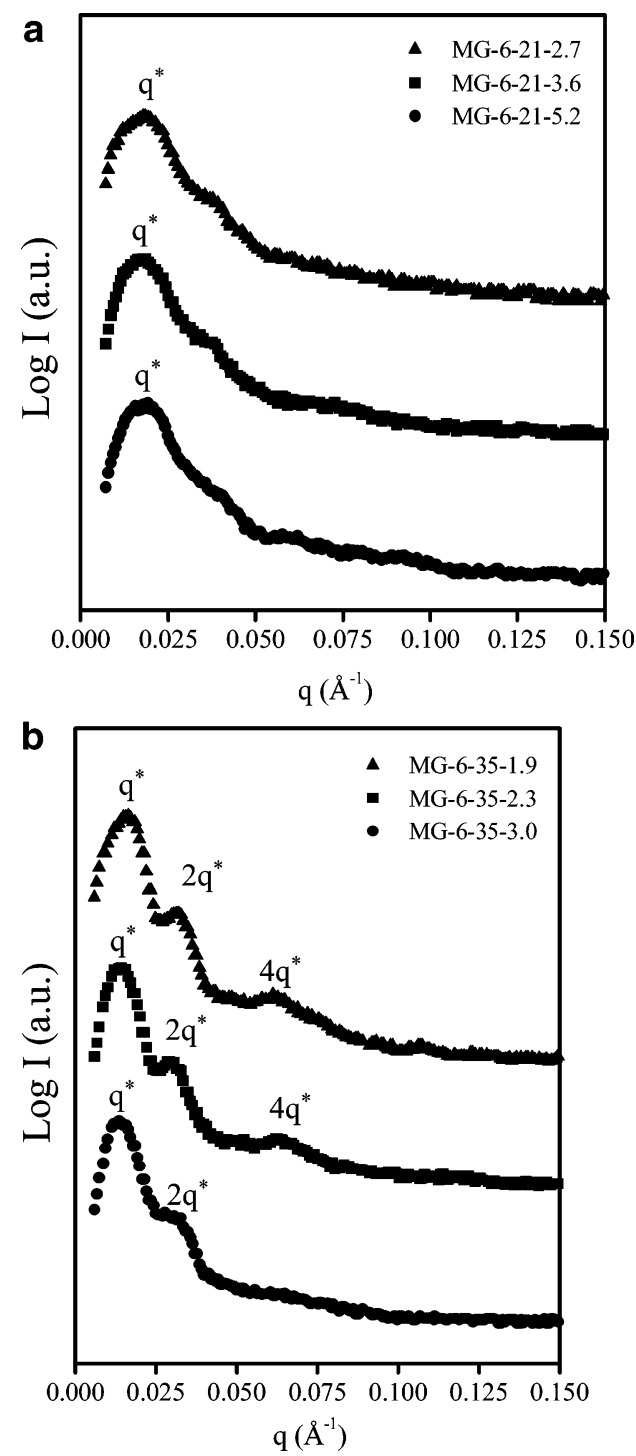

Figure 3. SAXS profiles of (a) MG-6-21 and (b) MG-6-35 materials.

points per molecule, which forms a fairly well ordered lamellar morphology. Thus, as the number of junction points per molecule increases, the ability to form well ordered morphologies is hindered, most likely due to the kinetic limitations imposed by large numbers of junction points and high molecular weight. The SAXS data in Figure 3a for all three fractions in MG-6-21 indicate a microphase-separated structure with a spacing of about $34.9 \mathrm{~nm}$. In the series of MG-6-35, the PS volume fraction places the material well into the lamellar region. The scattering profiles of these fractions, shown in Figure 3b, agree with the trend of decreasing long-range order with increasing number of junction points per molecule. This trend has also been found in all the trifunctional and tetrafunctional multigraft copolymer series. ${ }^{12,13,16}$ Morphological results for the multigraft copolymers with tri-, tetra-, and hexafunctional junction points can be found in Tables $1-3$.

Overall, the morphologies formed by all the series are the same as the predictions based on the constituting block copolymer concept and our general understanding of the morphologies of the miktoarm star constituting units. ${ }^{1,4,18-21}$ However, because of the differences in the functionality and asymmetry of junction points among these series, multigraft copolymers with same volume percent of PS do not necessarily form the same morphology.
Mechanical Properties. An overview of the mechanical properties of the multigraft copolymers is given in Table 4 . The stress - strain behavior of two tetrafunctional multigraft copolymers with about $20 \%$ PS compared to commercial thermoplastic elastomers (TPE), a Kraton 1102 and a Styroflex (BASF) copolymer, is shown in Figure 4. Kraton is a typical poly(styrene- $b$-isoprene- $b$-styrene) triblock copolymer forming hexagonally packed glassy PS cylinders in a rubbery PI matrix, while Styroflex is a poly[styrene- $b$-(styrene-co-butadiene)- $b$ styrene] (SBS) triblock copolymer with a styrene-co-butadiene copolymer as middle block. Molecular information on these two commercial materials and other two MG materials (MG-4-214.7 and MG-4-21-9.9) can be found in an early publication. ${ }^{16}$ Samples of these commercial TPE's were prepared and tested using the same procedures as employed with all the multigraft materials. Mechanical properties of such common TPE's like SBS triblock copolymers are strongly influenced by their architecture where the rubbery middle block forms bridges between the glassy PS domains, leading to a physical crosslinking which is generally reported as the reason for their excellent elastomeric properties. ${ }^{22}$ The requirements for this behavior are strongly segregated components with narrow interfaces and a sufficiently high molecular weight of the PS blocks to prevent pullout from the PS domains. In contrast to triblock copolymers, multigraft copolymers have a backbone with chemically different branches, and their morphology and properties can be tailored by changing molecular architecture. ${ }^{23}$ It was shown that mechanical properties of TPE's only show molecular weight dependence below $100 \mathrm{~kg} / \mathrm{mol}$, which is attributed to a lower degree of microphase segregation (represented by $N \chi$ ) at lower molecular weight. ${ }^{24}$ At $M_{\mathrm{n}}$ greater than $100 \mathrm{~kg} / \mathrm{mol}$ mechanical properties are insensitive to further increases in triblock molecular weight since the samples are all well within the strongly segregated regime. The multigraft copolymers used in this study have molecular weights far exceeding $100 \mathrm{~kg} / \mathrm{mol}$; thus, mechanical properties are expected to be independent of molecular weight.

As shown in Figure 4, Kraton and Styroflex reveal a higher tensile strength but distinctly lower strain at break than the tetrafunctional multigrafts. MG-4-21-9.9 shows a surprisingly high strain at break of about $1550 \%$, far exceeding the values for the commercial TPE's (Styroflex, 650\%; Kraton, 1080\%). The same high strain at break values could be observed for MG-4-14-3.5. This superelasticity is caused by their specific molecular architecture as schematically illustrated in Figure 5, where a rubbery PI backbone is anchored at multiple glass domains with two PS blocks at each junction point. ${ }^{16}$ This leads to enhanced physical cross-linking as compared to trifunctional multigraft copolymers or triblock copolymers. The enhanced mechanical properties of the multigraft materials arise from improved coupling of the highly elastic PI backbone to the reinforcing PS domains, resulting from having two or more PS chains at each junction point and a relatively large number of junction points per molecule.

Figure 6 shows the influence of the number of junction points on tensile strength and strain at break for tetrafunctional multigraft copolymers with about $20 \%$ PS. Tetrafunctional multigraft copolymers with fewer than five junction points show quite a low strain at break and low tensile strength. However, tensile strength and strain at break increase nearly linearly with increasing number of junction points. The same effect of linearly increasing tensile strength and increasing strain at break with increasing junction points per molecule was observed for hexafunctional multigraft copolymers. As hypothesized in a 
Table 4. Mechanical Properties of Tri-, Tetra- and Hexafunctional Multigraft Copolymers, Styroflex and Kraton

\begin{tabular}{|c|c|c|c|c|c|c|c|c|}
\hline \multirow[b]{2}{*}{ sample } & \multicolumn{2}{|c|}{ Young's modulus [MPa] } & \multicolumn{2}{|c|}{ yield stress [MPa] } & \multicolumn{2}{|c|}{ tensile strength [MPa] } & \multicolumn{2}{|c|}{ strain at break [\%] } \\
\hline & $E_{\mathrm{t}}$ & $\Delta^{a}$ & $\sigma_{y}$ & $\Delta^{a}$ & $\sigma_{\mathrm{t}}$ & $\Delta^{a}$ & $\epsilon_{\mathrm{b}}$ & $\Delta^{a}$ \\
\hline MG-3-17-3.7 & 1.96 & 1.55 & 0.08 & 0.07 & 6.39 & 1.63 & 1385.89 & 182.63 \\
\hline MG-3-17-2.6 & 1.03 & 0.71 & 0.08 & 0.04 & 5.06 & 1.14 & 1162.36 & 36.54 \\
\hline MG-3-44-3.8 & 180.3 & 6.35 & 4.44 & 0.25 & 9.75 & 0.91 & 792.82 & 54.08 \\
\hline MG-4-14-5.5 & 0.5 & 0 & 0.35 & 0.01 & 18.64 & 0.99 & 1472.76 & 2.88 \\
\hline MG-4-14-3.5 & 1.13 & 0.88 & 0.08 & 0.03 & 14.15 & 1.91 & 1549.78 & 74.93 \\
\hline MG-4-23-6.6 & 3.21 & 2.42 & 0.6 & 0.26 & 13.64 & 2.51 & 1329.54 & 107.71 \\
\hline MG-4-23-4.4 & 5.16 & 3.75 & 0.32 & 0.26 & 11.86 & 3.33 & 1272.78 & 202.26 \\
\hline MG-4-41-7.4 & 245.88 & 15.93 & 5.32 & 0.34 & 12.42 & 1.41 & 896.74 & 28.83 \\
\hline MG-4-41-5.3 & 261.55 & 5.57 & 5.72 & 0.15 & 12.76 & 0.71 & 898.36 & 29.73 \\
\hline MG-4-41-4.4 & 248.63 & 14.26 & 5.15 & 0.26 & 10.19 & 0.42 & 785.48 & 14.68 \\
\hline MG-6-21-5.2 & 16.06 & 4.04 & 0.79 & 0.27 & 14.63 & 3.46 & 1373.18 & 168.91 \\
\hline MG-6-21-3.6 & 26.98 & 7.95 & 0.98 & 0.19 & 12.24 & 1.1 & 1329.27 & 103.2 \\
\hline MG-6-21-2.7 & 29.27 & 9.03 & 1.39 & 0.12 & 11.03 & 1.08 & 1264.39 & 33.36 \\
\hline MG-6-35-3.0 & 140.33 & 11.76 & 3.41 & 0.19 & 8.31 & 0.53 & 881.46 & 38.58 \\
\hline Styroflex & 42.7 & 1.32 & 2.53 & 0.06 & 25.99 & 1.61 & 646.11 & 11.94 \\
\hline Kraton & 7.83 & 0.7 & 1.57 & 0.11 & 30.98 & 1.15 & 1078.41 & 25.34 \\
\hline
\end{tabular}

${ }^{a}$ Standard deviation.

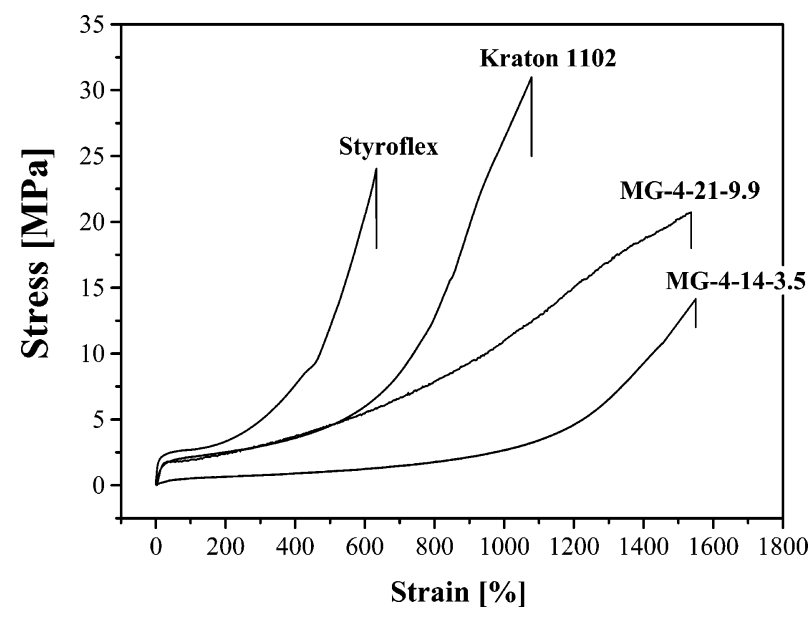

Figure 4. Stress-strain behavior of tetrafunctional multigraft copolymers compared to commercial TPE's.



Figure 5. Chain conformation of tetrafunctional multigraft copolymers in microphase-separated state.

previous study, ${ }^{16}$ a linear dependence of mechanical properties of the number of junction points was observed, and a high number of junction points was found to result in enhanced material properties. The long-range order of the morphology of multigraft copolymers decreases with increasing number of junction points, as shown previously ${ }^{13}$ in a study of tetrafunctional multigraft copolymers. Thus, contrary to previous understanding, resulting from work on SBS triblock copolymers, ${ }^{24}$ a well ordered morphology is not necessary to achieve good mechanical properties. The mechanical properties of multigraft copolymers are influenced by the number of junction points (n) since an increasing number of junction points is connected with enhanced physical cross-linking. The existence of smaller grain sizes (lower long-range order) at a high number of junction points per molecule can also be correlated with an improved
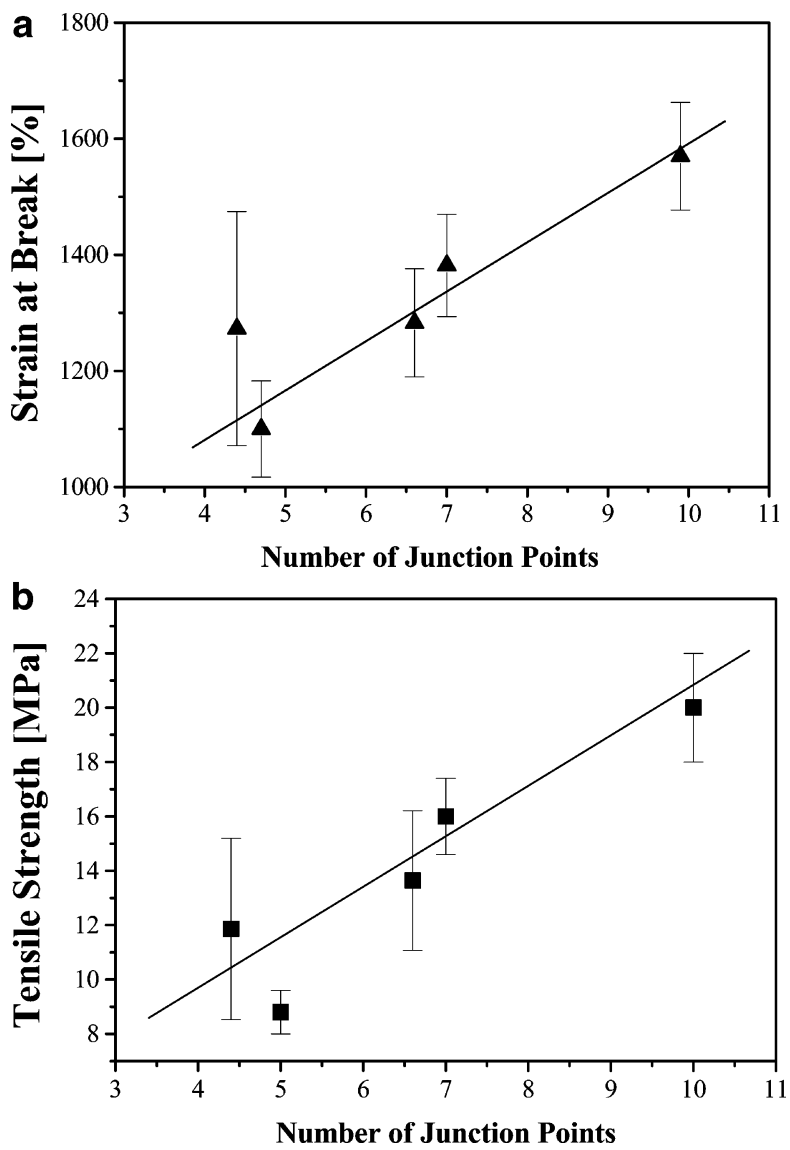

Figure 6. (a) Influence of number of branch points on (a) strain at break; (b) tensile strength of tetrafunctional multigraft copolymers with about $20 \%$ of PS.

property profile as earlier shown in the case of weakly segregated block copolymers. ${ }^{25-27}$

As the PS content in tetrafunctional $(n=4)$ multigraft copolymers with about four junction points $(\beta=4)$ increase from $14 \%$ to $41 \%$, the morphology changes from PS spheres, to PS cylinders, to lamellae. This results in a transition from elastomeric to thermoplastic behavior, as indicated by Figure 7a. The glassy PS domains in the lamellae are deformed by yielding during the loading process, causing a distinct yield point and the transition to thermoplastic behavior. There is a significant increase in yield stress and a strong decrease in strain with increasing PS content but without a corresponding increase 

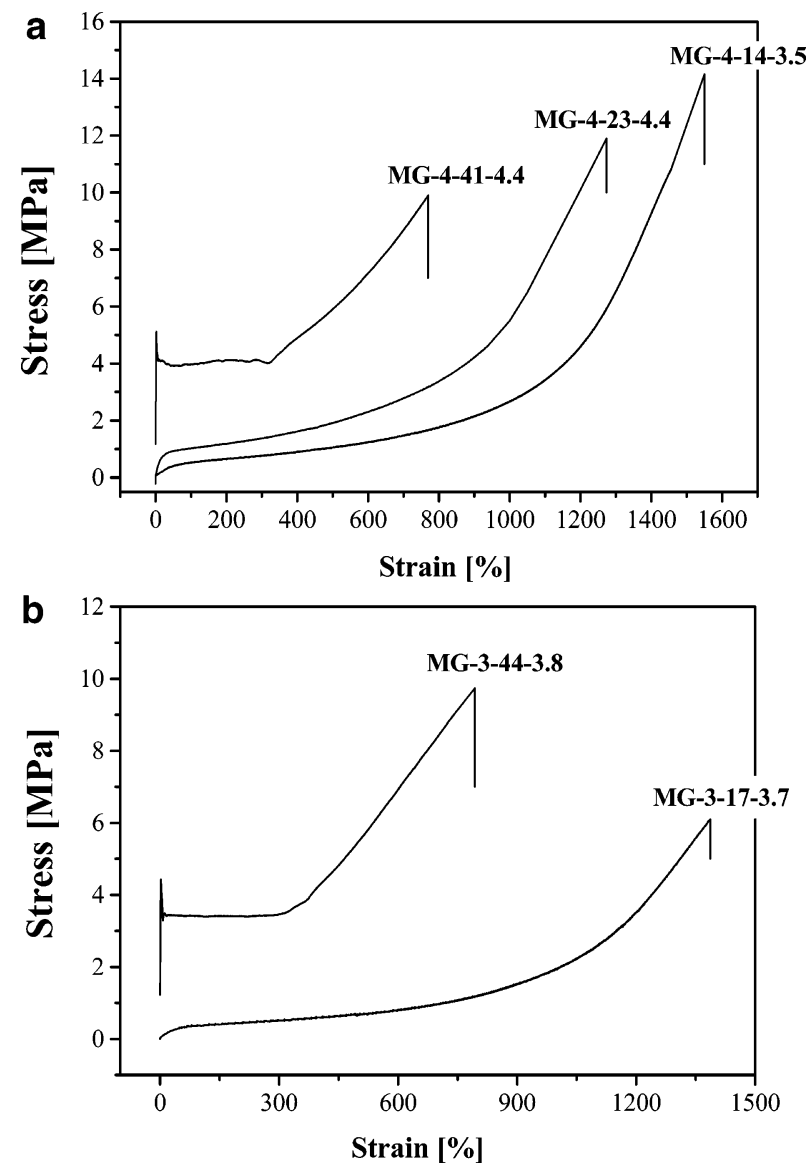

Figure 7. Influence of PS content on tensile properties of multigraft copolymers with about four junction points. (a) Tetrafunctional MG materials: MG-4-14-3.5 PS spheres; MG-4-23-4.4 PS cylinders; MG-4-41-4.4 lamellae. (b) Trifunctional MG materials: MG-3-17-3.7 PS spheres; MG-3-44-3.7 lamellae.

in tensile strength. The same behavior could be found for hexafunctional $(n=6)$ multigraft copolymers with about three junction points (Table 4). In contrast, trifunctional $(n=3)$ multigraft copolymers with about four junction points show a distinct increase in tensile strength with increasing PS content, which is typical for TPE's (Figure 7b). The transition from elastomeric to thermoplastic behavior caused by a change in morphology from spherical or cylindrical to lamellar structures is clearly in agreement with linear SBS or SIS triblock copolymers.

The influence of junction point functionality on mechanical properties of multigraft copolymers with a PS content of about $20 \%$ is shown in Figure 8. In contrast to common TPE's, which usually have same the morphologies at similar volume fractions, the multigraft copolymers compared in Figure 8 have different morphologies at similar volume fraction resulting from changes in the constituting block copolymer asymmetry (ratio of A to $\mathrm{B}$ arms) in tri-, tetra-, and hexafunctional multigraft copolymers. While strain at break is less influenced by functionality, the tensile strength of the cylinder forming tetrafunctional multigraft is about the twice that of trifunctional material of the same composition but with a spherical morphology (Figure 8a). As reported previously, ${ }^{28}$ there is an increased density of molecular interactions between the PS nanodomains in tetrafunctional multigraft copolymers compared to trifunctional multigraft copolymers, which was revealed by modeling of chain dynamics, leading to enhanced mechanical properties. Hexafunctional multigraft materials with lamellar morphology (MG-6-21-3.6) show even a higher tensile strength, suggesting new possibilities
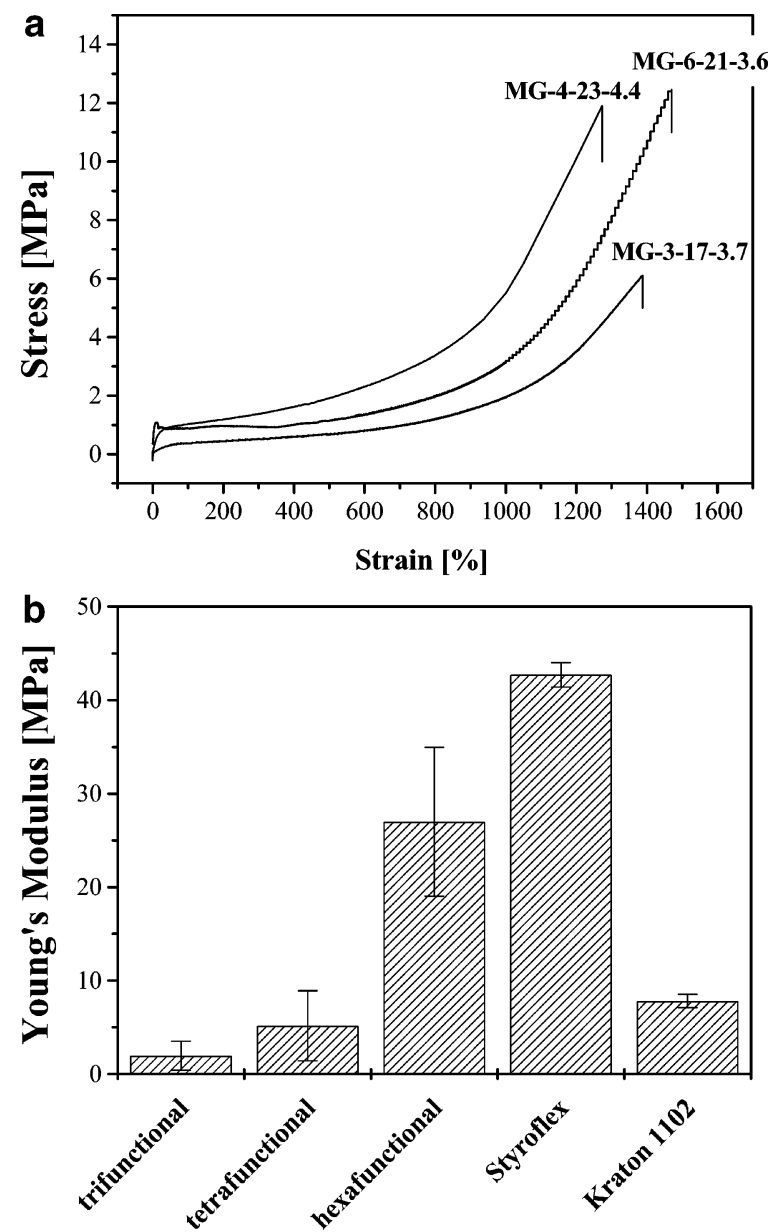

Figure 8. Influence of functionality on (a) stress - strain behavior and (b) Young's modulus of multigrafts with a PS content of about $20 \%$ and four junction points: MG-3-17-3.7 PS spheres; MG-4-23-4.4 PS cylinders; MG-6-21-3.6 lamellae.

for design of TPE's with high levels of strain at break. The large tensile strength combined with large strain at break is a surprising result for materials with lamellae morphology; highly elastic TPE's exhibiting large strains at break generally have cylindrical or spherical morphologies with rubbery matrices. It is worth noting that in linear SIS triblocks commonly used as TPE's that the lamellar morphology is usually formed at $35 \%-$ $65 \%$ of PS. In the hexafunctional multigrafts of this study, the unique molecular architecture allowed the formation of a lamellar structure at about $20 \%$ PS, resulting in the distinct yield point shown in Figure 8a. The elastomeric property profile of hexafunctional multigraft copolymers with lamellar morphology may be caused by the much smaller width of the PS layers compared to the rubbery PI layers, where the PS layers act as reinforcing domains. Figure 8 demonstrates the opportunity of tailoring mechanical properties in a wide range by changing molecular architecture.

Besides large tensile strength and elasticity, a low Young's modulus is required for successful application of TPE's. The Young's modulus of hexafunctional copolymers is significantly higher compared to tri- and tetrafunctional multigrafts, as shown in Figure 8b, and also far exceeds the values of commercial Kraton. This shows that the multigraft materials with hexafunctional junction points provide significantly enhanced mechanical reinforcement compared to the tri- or tetrafunctional materials. Another reason for the large Young's modulus of hexafunctional multigraft copolymers is the lamellae morphology observed for materials with about $20 \%$ PS compared to tri- or tetrafunctional 




Figure 9. Stress-strain behavior of MG-3-17-3.7 and MG-4-14-3.5 copolymers; both have a spherical morphology with PS as the minority domains.

multigraft copolymers comprised of a rubbery matrix with glassy domains. However, trifunctional and tetrafunctional multigraft copolymers show lower Young's modulus values compared to Kraton 1102, as shown in Figure 8b.

As already observed, the morphology of multigraft copolymers depends on the functionality at constant PS content. This change in morphology can be attributed to a change in the molecular asymmetry of the constituting block copolymer units as a result of changing the ratio of PS and PI arms linked at each junction point. ${ }^{4,18-21}$ This molecular architecture driven morphological change is found to produce a pronounced change in mechanical properties at similar overall composition and molecular weight. To separate the influence of molecular architecture on tensile properties from the influence of changes in morphology, two multigraft copolymers with different architecture but the same morphology are compared. Figure 9 shows stress-strain curves for MG-3-17-3.7 and MG-4-14-3.5. Both materials have similar numbers of junction points per molecule (3.5-3.7), and both have a spherical PS morphology. Results shown in Figure 9 demonstrate the superior performance of the tetrafunctional multigraft copolymer over the trifunctional. Both the strain at break and tensile strength of the tetrafunctional multigraft copolymer are significantly higher. This occurs despite the fact that the tetrafunctional material possesses a slightly lower PS content and a slightly lower average number of junction points per molecule. The enhanced interaction of the PS branches for tetra- and hexafunctional multigraft copolymers, in which multiple PS arms per molecule are tied into each PS hard domain, causes an effective physical multicross-linking which is more effective than the single-chain physical cross-linking in trifunctional multigraft copolymers and SIS triblock copolymers.

As shown in Figure 10, the tensile properties of multigraft copolymers with about $40 \%$ of PS are much less influenced by functionality than in materials with lower PS content. Lamellar morphologies were formed for all three functionalities at this composition, and compared with multigrafts with lower PS content all these samples showed a decrease in strain at break. Young's modulus values about 10 times higher are observed for multigrafts with PS contents of about $40 \%$ compared to multigrafts with $20 \%$ PS; the tetrafunctional copolymers show the highest values (Table 4). Comparing Figures 8a and 10, the tensile strength is seen to increase for trifunctional multigrafts but decrease for tetra- and hexafunctional multigraft copolymers with increasing PS content. The molecular architecture does not appear to influence the mechanical properties much in the composition range of $35 \%-45 \%$ of PS, where a lamellar

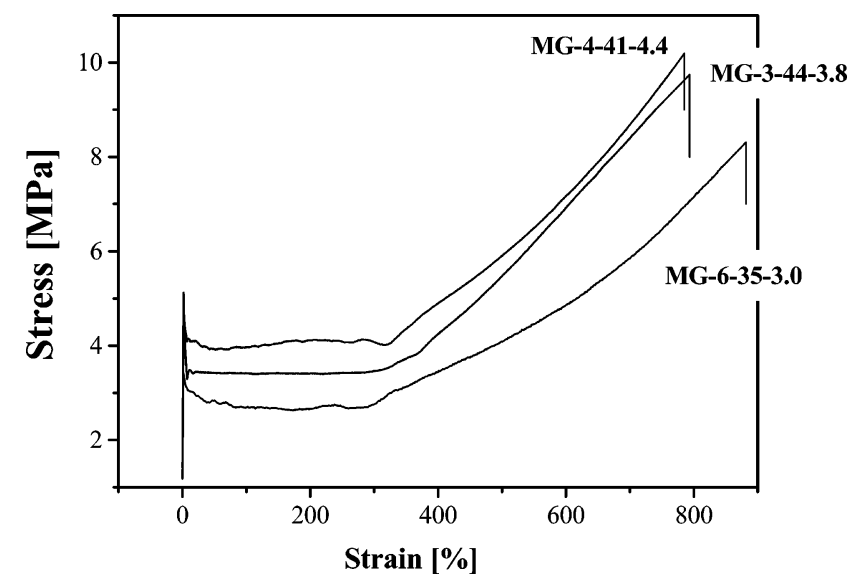

Figure 10. Influence of junction point functionality on stress-strain behavior of multigrafts with about $40 \%$ of PS content.

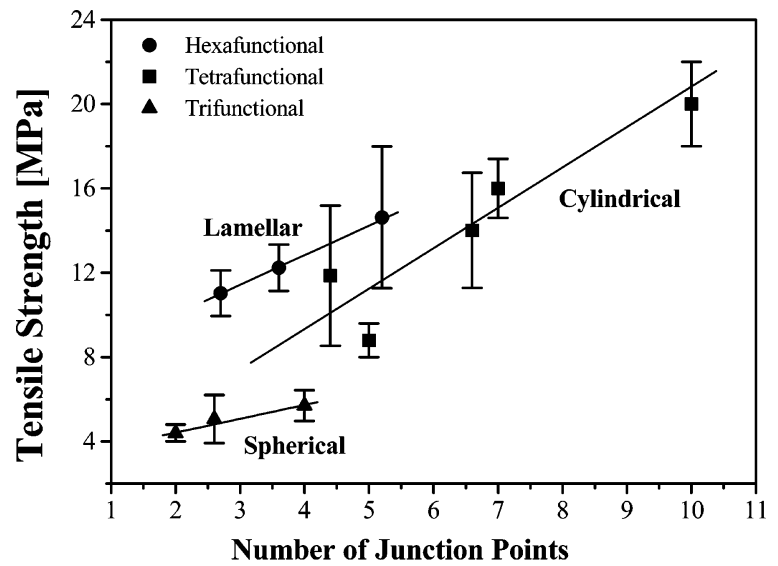

Figure 11. Influence of number of junction points on tensile strength for tri-, tetra-, and hexafunctional multigraft copolymers at about $20 \%$ of PS.

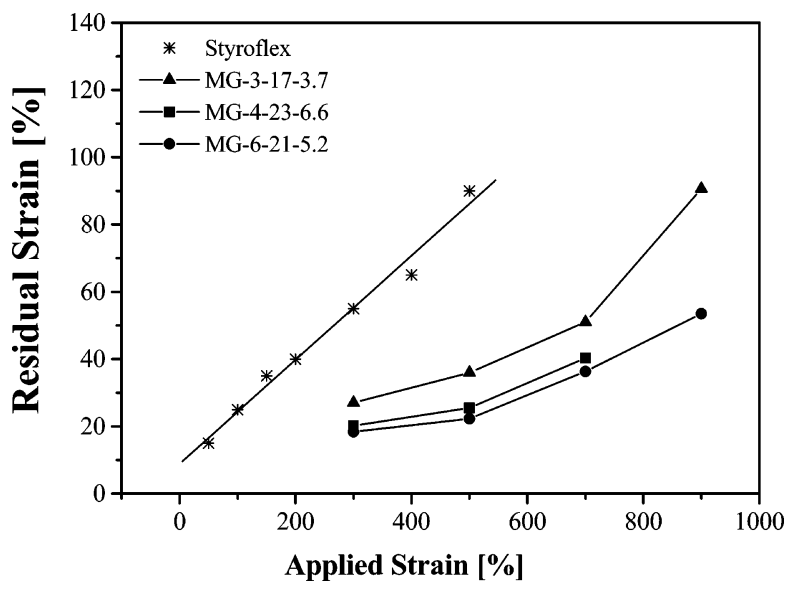

Figure 12. Residual strain in hysteresis experiments of multigraft copolymers compared to Styroflex.

morphology and thermoplastic behavior consistent with yielding of the lamellae are observed.

Figure 11 shows the combined influence of morphology and number of junction points on tensile strength of multigraft copolymers with about $20 \%$ PS. In this diagram the volume percent of PS is constant, but as the number of junction points changes both branch point functionality and morphology are varying. Trifunctional multigrafts with spherical structure reveal low tensile strength, which does not increase much with increasing number of junction points. The slopes of the tensile 


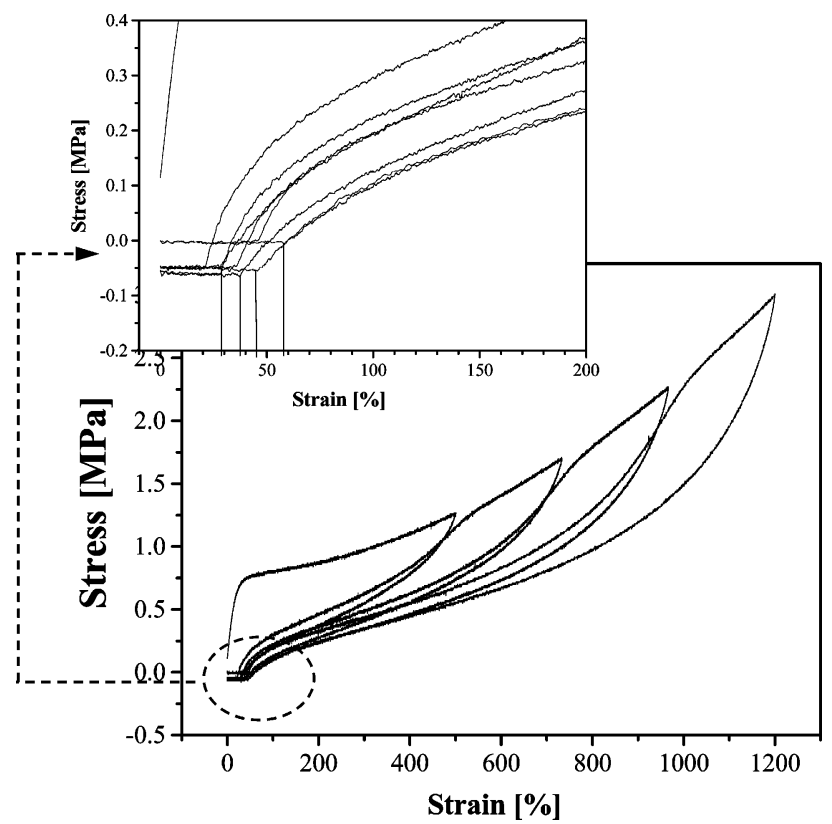

Figure 13. Incremental hysteresis experiment of a tetrafunctional multigraft copolymer MG-4-23-6.6.

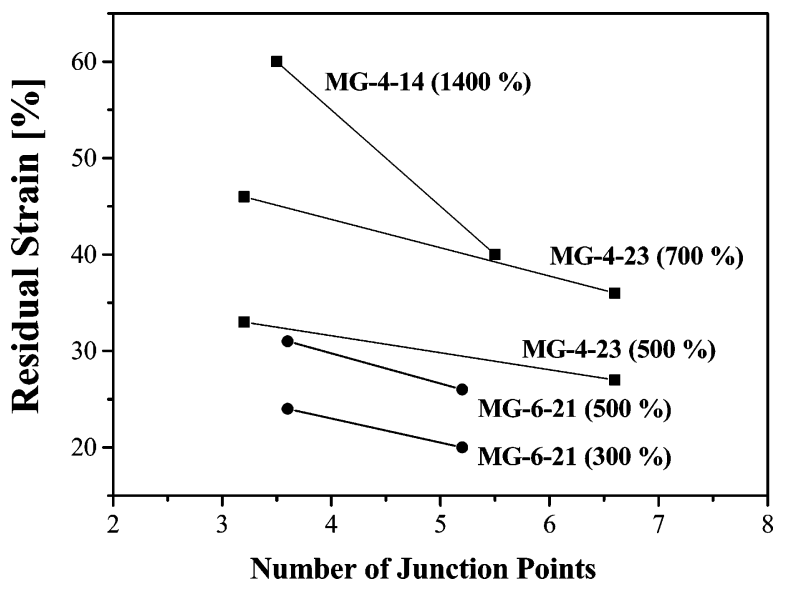

Figure 14. Residual strain in hysteresis experiments of tetra- and hexafunctional multigraft copolymers at varying deformation dependent on the number of junction points.

strength vs junction points curves for hexafunctional and tetrafunctional multigrafts are similar. And, in the range of low number of junction points, higher strengths are observed for the hexafunctional multigraft materials. Both tetra- and hexafunctional MG materials reveal a much greater increase in tensile strength with increasing number of junction points as compared to the trifunctional multigrafts.

The results of hysteresis experiments at 300\%-900\% deformation, shown in Figure 12, demonstrate the high elasticity of these multigrafts copolymers compared to commercial Styroflex. For the multigrafts, residual strain does not change much with increasing applied strain. For Styroflex, with a linear molecular architecture and a similar morphology, however, a much greater increase in residual strain with increasing applied load is observed. Hexafunctional copolymers with $21 \%$ PS and 5.2 junction points (MG-6-21-5.2) show very high elasticity with a residual strain of about $55 \%$ after an applied strain of $900 \%$, a level of strain at which common commercial TPE's would have already failed. Recently, we have found ${ }^{29}$ that tetrafunctional multigraft copolymers can be strained up to $1400 \%$ while showing a residual strain of only about $40 \%$. Incremental hysteresis experiments have been carried out for a tetrafunctional multigraft copolymer MG-4-23-6.6, as shown in Figure 13. Despite repeated loading of the material, the residual strain is very low up to a deformation of $1200 \%$. With increasing number of junction points per molecule, the residual strain observed in first hysteresis experiments decreases for tetra- and hexafunctional multigrafts, indicating a dependence of elasticity on number of junction points (Figure 14). Further hysteresis studies are necessary to confirm these observations.

\section{Conclusions}

Two new parameters which have distinct influence on the mechanical properties of multigraft copolymers with total PS contents between $14 \%$ and $23 \%$ are found in our investigations: (1) Functionality of the grafting points, which influences the morphology and thus the mechanical properties of multigraft copolymers. An increasing functionality causes a change in morphology according to the Milner phase diagram, resulting in a high level of tensile strength for tetrafunctional (cylinder) and hexafunctional (lamellae) multigraft copolymers, about twice that of the trifunctional multigrafts with spherical morphology. (2) Number of junction points per molecule. With increasing number of junction points, the grain size of the microphase-separated morphology decreases and strain at break and tensile strength increase. A linear increase in the mechanical properties (strength and elongation at break) on the number of junction points is found. Tetrafunctional multigraft copolymers show a surprisingly high strain at break, far exceeding that of commercial TPE's. Hysteresis experiments at about $300 \%-900 \%$ deformation have demonstrated the high elasticity of hexa- and tetrafunctional multigraft copolymers compared to commercial TPE's like Kraton or Styroflex. At high PS contents from $35 \%$ to $45 \%$ multigraft copolymers loose their elastomeric property profile, and the mechanical properties are insensitive to junction point functionality.

Acknowledgment. This work was supported by the U.S. Army Research Office (ARO) under Contract W911NF-04-10329. We also acknowledge the use of central facility and the W.M. Keck Electron Microscopy in the Material Research Science and Engineering Center (MRSEC) at the University of Massachusetts. R. Weidisch and U. Staudinger acknowledge financial support from German Science Foundation (DFG). A portion of this research was conducted at the Center for Nanophase Materials Sciences, which is sponsored at Oak Ridge National Laboratory by the Division of Scientific User Facilities, U.S. Department of Energy.

\section{References and Notes}

(1) Matsen, M. W.; Bates, F. S. Macromolecules 1996, 29, 1091.

(2) Matsen, M. W.; Schick, M. Macromolecules 1994, 27, 4014.

(3) Matsen, M. W.; Schick, M. Macromolecules 1994, 27, 187.

(4) Milner, S. Macromolecules 1994, 27, 2333.

(5) Matsen, M. W.; Thompson, R. B. J. Chem. Phys. 1999, 111, 7193.

(6) Pochan, D. J.; Gido, S. P.; Pispas, S.; Mays, J. W.; Ryan, A. J.; Fairclough, J. P.; Hamley, I. W.; Terrill, N. Macromolecules 1996, 29, 5091.

(7) Gido, S. P.; Lee, C.; Pochan, D. J.; Pispas, S.; Mays, J. W.; Hadjichristidis, N. Macromolecules 1996, 29, 7022.

(8) Lee, C.; Gido, S. P.; Pitsikalis, M.; Mays, J. W.; Beck-Tan, N.; Trevino, S. F.; Hadjichristidis, N. Macromolecules 1997, 30, 3732.

(9) Lee, C.; Gido, S. P.; Poulos, Y.; Hadjichristidis, N.; Beck Tan, N.; Trevino, S. F.; Mays, J. W. J. Chem. Phys. 1997, 107, 6460.

(10) Gido, S. P.; Wang, Z. G. Macromolecules 1997, 30, 6771

(11) Lee, C.; Gido, S. P.; Poulos, Y.; Hadjichristidis, N.; Beck Tan, N.; Trevino, S. F.; Mays, J. W. Polymer 1998, 39, 4631. 
(12) Xenidou, M.; Beyer, F. L.; Hadjichristidis, M.; Gido, S. P.; Beck Tan, N. Macromolecules 1998, 31, 7659.

(13) Beyer, F.; Gido, S. P.; Buschl, C.; Iatrou, H.; Uhrig, D.; Mays, J. W.; Chang, M.; Garetz, B. A.; Balsara, N.; Beck Tan, N.; Hadjichristidis, N. Macromolecules 2000, 33, 2039.

(14) Zhu, Y.; Weidisch, R.; Gido, S. P.; Velis, G.; Hadjichristidis, N. Macromolecules 2002, 35, 5903.

(15) Olvera de la Cruz, M.; Sanchez, I. C. Macromolecules 1986, 19 , 2501.

(16) Weidisch, R.; Gido, S. P.; Uhrig, D.; Iatrou, H.; Mays, J.; Hadjichristidis, N. Macromolecules 2001, 34, 6333.

(17) Uhrig, D.; Mays, J. W. Macromolecules, 2002, 35, 7182.

(18) Zhu, Y.; Gido, S. P.; Moshakou, M.; Iatrou, H.; Hadjichristidis, N.; Park, S.; Chang, T. Macromolecules 2003, 36, 5719.

(19) Beyer, F. L.; Gido, S. P.; Uhrig, D.; Mays, J. W.; Beck Tan, N.; Trevino, S. F. J. Polym. Sci., Part B: Polym. Phys. 1999, 37, 3392.

(20) Beyer, F. L.; Gido, S. P.; Poulos, Y.; Avgeropoulos, A.; Hadjichristidis, N. Macromolecules 1997, 30, 2373.

(21) Yang, L.; Hong, S.; Gido, S. P.; Velis, G.; Hadjichristidis, N. Macromolecules 2001, 34, 9069.
(22) Holden, G.; Legge, N. R. In Thermoplastic Elastomers; Legge, N. R., Holden, G., Quirk, R., Schroeder, H. E., Eds.; Hanser: Muinch, 1987; pp 47-70.

(23) Kennedy, J. P. In Thermoplastic Elastomers; Legge, N. R., Holden, G., Quirk, R., Schroeder, H. E., Eds.; Hanser: Muinch, 1996; pp 365394.

(24) Quirk, R. P.; Morton, M. In Thermoplastic Elastomers; Legge, N. R., Holden, G., Quirk, R., Schroeder, H. E., Eds.; Hanser, Munich, 1996; pp 71-100.

(25) Weidisch, R.; Stamm, M.; Michler, G.; H.; Fischer, H.; Jerome, R. Macromolecules 1999, 32, 742.

(26) Weidisch, R.; Michler, G. H.; Arnold, M.; Fisher, H. J. Mater. Sci. 2000, 35, 1257.

(27) Weidisch, R.; Michler, G. H. In Block Copolymers; Balta Calleja, F. J., Roslaniec, Z., Eds.; Marcel Dekker: New York, 2000; pp 215250.

(28) Sumpter, B. G.; Mays, J. W.; Noid, D. W.; Gido, S. P.; Weidisch, R. Polym. News 2004, 29, 302

(29) Staudinger, U.; Weidisch, R.; Zhu, Y.; Gido, S. P.; Uhrig, D.; Mays, J.; Iatrou, H.; Hadjichristidis, N. Macromol. Symp. 2006, 233, 42.

MA060067S 